\title{
Simulation Studies for a Surface Veto Array to Identify Astrophysical Neutrinos at the South Pole
}

\author{
The IceCube-Gen2 Collaboration ${ }^{\dagger}$ \\ ${ }^{\dagger}$ http://icecube.wisc.edu/collaboration/authors/icrc15_gen2 \\ E-mail: sebastian.euler@icecube.wisc.edu, \\ jgonzalez@icecube.wisc.edu, bsr25@uclive.ac.nz
}

\begin{abstract}
Motivated by the discovery of high-energy astrophysical neutrinos with IceCube, we study the prospects for improved measurements of neutrinos of astrophysical origin with a surface array combined with IceCube or a next generation neutrino detector at the South Pole. Backgrounds in astrophysical neutrino searches are reduced by tagging muons and neutrinos of atmospheric origin by detecting the accompanying air shower. We consider various air shower array configurations, including different array layouts and detector station sizes, and study their air shower detection efficiency. We will report on the various approaches we have used to understand the capabilities of such arrays.
\end{abstract}

Corresponding authors: S. Euler ${ }^{* 1}$, J. Gonzalez ${ }^{2}$, B. Roberts ${ }^{3}$

${ }^{1}$ Dept. of Physics and Astronomy, Uppsala University, Uppsala, Sweden

${ }^{2}$ Bartol Research Institute, University of Delaware, USA

${ }^{3}$ Dept. of Physics and Astronomy, University of Canterbury, Christchurch, New Zealand

The 34th International Cosmic Ray Conference,

30 July- 6 August, 2015

The Hague, The Netherlands

${ }^{*}$ Speaker. 


\section{Introduction}

A crucial technique in the IceCube discovery of astrophysical neutrinos [2] was to use the outer layers of the detector as an active veto against penetrating muons. This approach, however, effectively reduces the fiducial volume of the detector by about $50 \%$, and requires an additional cut on the observed charge, corresponding to an energy threshold of $30 \mathrm{TeV}$. An alternative possibility would be a dedicated veto array on the ice surface, tagging atmospheric muons by detecting the accompanying air shower. This approach would make it possible to use the full detector as target material, and in searches for muon neutrinos even the ice above the detector, since high-energy muons can reach the detector after being created

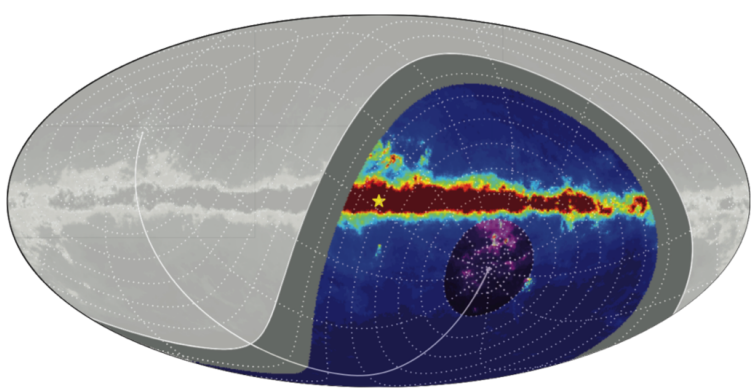

Figure 1: Skymap in galactic coordinates of a GALPROP $\pi^{0} 1.1 \mathrm{TeV}$ simulation. The colored area shows the approximate region of the sky covered by the veto arrays studied in this work, the purple region indicates coverage with the IceTop array. The yellow star marks the Galactic Center, the dark gray band the remaining region above the horizon that is not covered by the surface veto. Plot taken from [1]. several kilometers away. In fact, IceTop, the surface component of the IceCube Neutrino Observatory [3], is used as a veto array in several analyses [4, 5, 6], although its limited size covers only a small solid angle of the upper hemisphere (see Fig. 1). The prospects for a larger array are particularly interesting in the context of the planned extension IceCube-Gen2 [7]. If possible, a surface veto should be large enough to allow the observation of the Galactic Center and have an energy threshold that is low enough to study the astrophysical neutrino flux at lower energies than to date. A summary of motivations and techniques for such a surface array can be found in [8].

The performance of a prospective array and its optimal geometry have to be estimated by simulations. Ideally, a full simulation should be generated, covering all aspects from the interaction of the cosmic ray in the atmosphere to the detector response including a simulation of the electronics. Such a full simulation is however very resource-intensive and cannot be done with high statistics for a large number of configurations, as desired in a design study.

Therefore, we follow two complementary approaches in parallel. We have developed a fast Monte Carlo simulation based on lateral distribution functions (section 2). This simulation uses only average shower properties, even though for a highly efficient veto exceptional showers become important. Nonetheless, with this approach we obtain a reasonable estimate of the performance of a large number of different array configurations within a short time. These simulations can guide the design process.

The parallel approach is a simulation chain based on detailed simulations of extensive air showers using CORSIKA [9], which includes simulating their interaction with various detector array configurations (section 3). While this approach is more resource-intensive, it allows the study of rare air showers. Examples are showers in which the primary cosmic ray interacts very deep in the atmosphere, or production of highly energetic muons in exotic processes. In this case 
the footprint of the air shower, and therefore the detection probability on the surface, can be very small while still producing muons that reach IceCube. This approach enables the study other exotic processes that could produce a background.

\section{LDF-based Monte Carlo simulation}

\subsection{Lateral Distribution Functions}

The basis of the simulation described here are lateral distribution functions - empirical parameterizations of air showers - which describe the particle density in an air shower as a function of the lateral distance to the shower core.

An important aspect of a detector at the South Pole is that with time it will be gradually buried by drifting snow. The IceTop array observes attenuation of the electromagnetic component of the air showers, getting stronger with time [3]. A future surface veto array must therefore be either efficient enough to rely on the muonic component only, or constructed such that it is not buried by snow (e.g. on stilts above the surface). Therefore, we use separate formulations for the density of electrons and muons, taken from [10]. Their sum is equivalent to the classic formulation by Greisen [11]. Fig. 2a shows the individual functions for muons and electrons and their sum, together with the Greisen curve for a $300 \mathrm{TeV}$ shower at the altitude of the South Pole (2835 m). It should be noted that the parametrizations used are quite old and will be superseded by more recent results from IceTop [12,13] in the future.

While this approach could in principle also be applied to heavier primaries, the results presented here assume pure proton primaries. On the other hand, the approach by construction ignores variations in the shower development. Rare showers, where for example a large fraction of the primary energy is transferred to a single muon, cannot be simulated with this setup. Such events become important for a high-efficiency veto, and more detailed simulations are needed (see section 3).

\subsection{Detection efficiency}

To obtain the air shower detection efficiency for a given array geometry, the LDFs are evaluated at the positions of the simulated detector stations. From the particle density, a Poissonian probability to see a "hit" in each station is calculated, taking into account the station's surface area. Fig. $2 \mathrm{~b}$ shows the footprint of a simulated $300 \mathrm{TeV}$ shower with a zenith angle of $8^{\circ}$ on the IceTop detector. With increasing shower inclination, the thickness of the detectors becomes important: the sensitive area of thin detectors (e.g. scintillator panels) is much smaller for very inclined showers than for vertical ones. More spherical detectors (e.g. water/ice Cherenkov tanks) have a more uniform sensitivity. This effect is also taken into account in the simulation. ${ }^{1}$

For a given energy and zenith angle, many similar showers are simulated. To emulate the conditions of a dedicated veto array and to save computation time, zenith angle and radial position are chosen in conjunction, such that high-energy muons from the shower pass through IceCube.

\footnotetext{
${ }^{1}$ This disadvantage of scintillation detectors can be partly compensated by tilting the panels increasingly with radius. This technique restores the apparent size for inclined showers, at least for detector stations close to the shower axis, but is currently not taken into account in the simulation.
} 


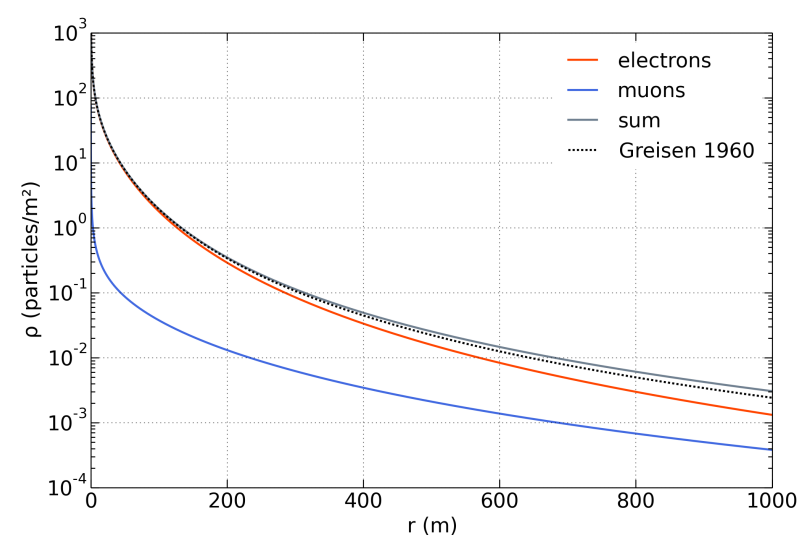

(a) Lateral Distribution Functions

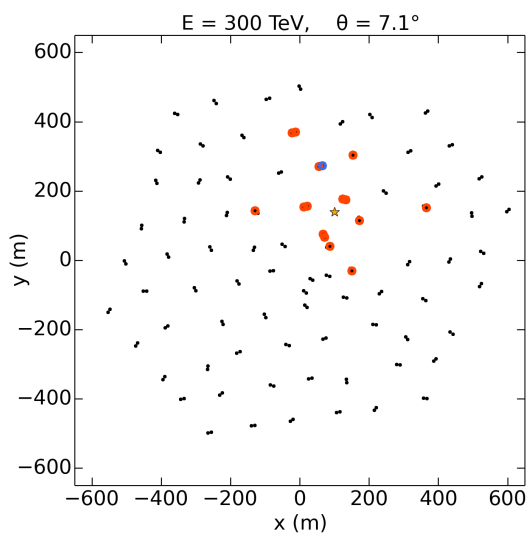

(b) Simulated example shower

Figure 2: LDFs used in this study and the simulated footprint of a $300 \mathrm{TeV}$ shower on the IceTop array. The tank marked in blue has registered a muon, tanks in red have detected the electromagnetic part of the shower. The orange star marks the surface position of the shower core.

For each shower, the whole array is considered "triggered" if at least one station has seen a hit. The detection efficiency is then the ratio of triggered showers to all simulated showers.

\subsection{Geometries}

Three example geometries are studied in detail here. The first (Fig. 3) is an extension of the existing IceTop array, called IceVeto [1]. It consists of IceTop in the center and 943 additional detector stations, each of the same size as one original IceTop tank. The new detectors are arranged in concentric rings around IceTop. In order to achieve a detection efficiency that does not depend strongly on the inclination of the air shower, while using as few tanks as possible, the distance between the rings increases with radius, while the distance between tanks along the same ring stays approximately the same. The array has a maximum radius of $6.7 \mathrm{~km}$, corresponding to a zenith angle as seen from IceCube of $\theta \approx 75^{\circ}$, and thus covers the ele-

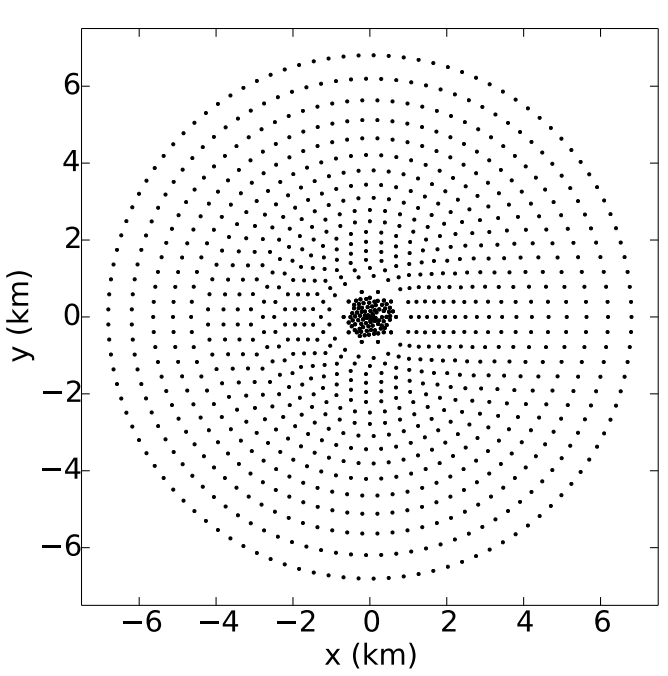

Figure 3: The IceVeto geometry. vation of the Galactic Center at $\theta=61^{\circ}$.

The other two geometries are simpler designs. In these cases, the array covers a square area with a side of $14 \mathrm{~km}$, such that for IceCube or a next-generation detector in the center, the array is again large enough to cover the elevation of the Galactic Center. The individual stations are 


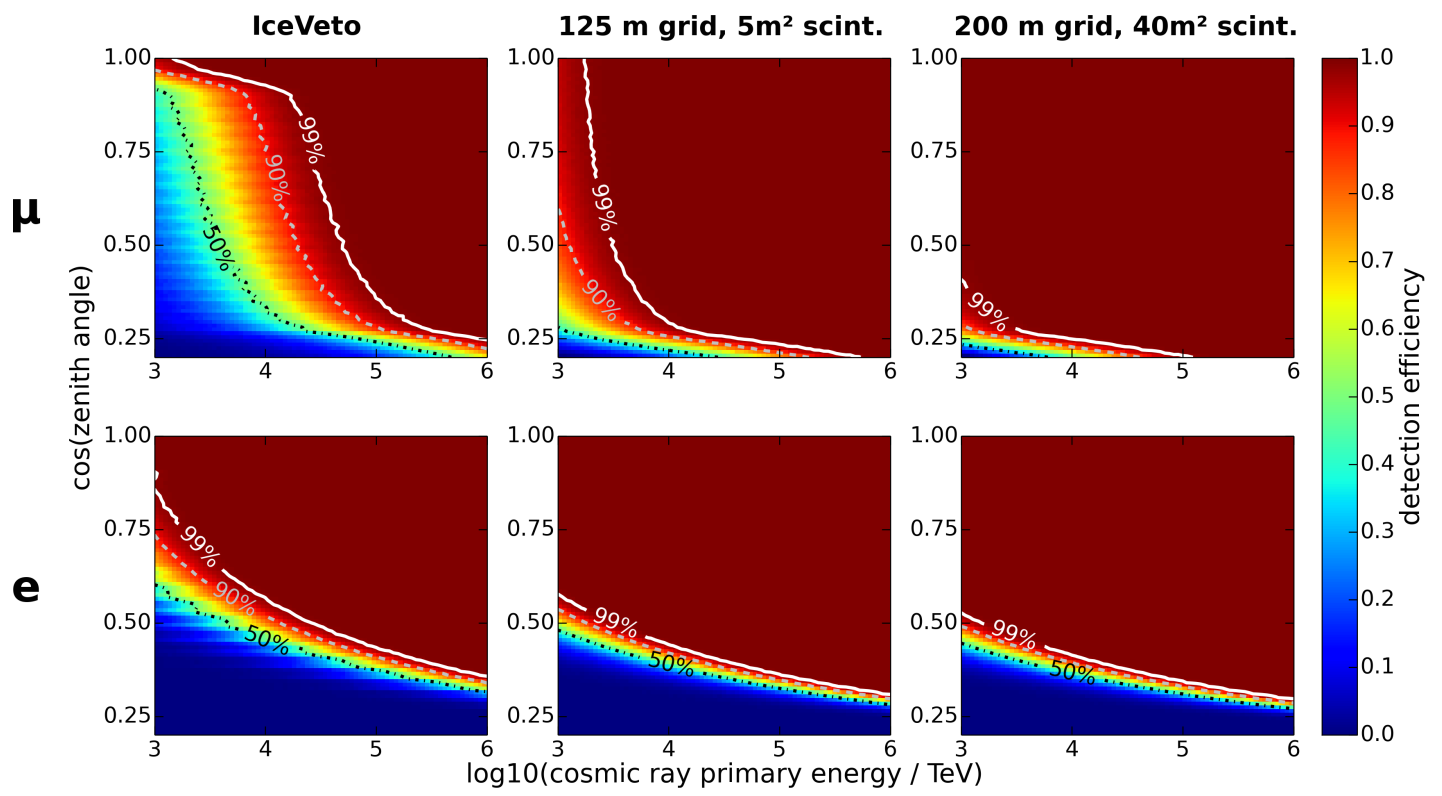

Figure 4: Detection efficiencies for IceVeto (left), the $125 \mathrm{~m}$ grid geometry (middle), and the $200 \mathrm{~m}$ grid geometry (right), separate for muons (top) and the electromagnetic shower component (bottom). The lines indicate detection efficiencies of 99\% (white, solid), 90\% (gray, dashed), and $50 \%$ (black, dot-dashed).

arranged on a regular rectangular grid. Two different combinations of detector area and station spacing are studied. The first variant has a dense grid with a spacing of $125 \mathrm{~m}$, resulting in a total number of roughly 12500 stations. Each station consists of a $5 \mathrm{~m}^{2}$ scintillator panel of $1 \mathrm{~cm}$ thickness. An important parameter of a surface array is the fill factor, the ratio of sensitive area to total surface area. This design achieves a fill factor of $3.2 \times 10^{-4}$. The IceVeto geometry, for comparison, has a total fill factor of about $2 \times 10^{-5}$, albeit with a radially varying density. The second grid variant has a larger spacing of $200 \mathrm{~m}$, resulting in 4900 stations, but assumes extremely large scintillator panels with an area of $40 \mathrm{~m}^{2}$ to achieve a fill factor of $1 \times 10^{-3}$.

\subsection{Results}

The detection efficiency is calculated at 6000 test points on a grid of primary energies between $1 \mathrm{PeV}$ and $1 \mathrm{EeV}\left(3.0<\log \left(E_{\text {prim }} / \mathrm{TeV}\right)<6.0\right)$ and zenith angles between $0^{\circ}$ and $78^{\circ}$ $(1.0>\cos (\theta)>0.2)$. For each point in this parameter space, 10000 air showers were simulated. Fig. 4 shows the resulting detection efficiency maps as function of zenith angle and primary proton energy, together with the contour lines of $99 \%, 90 \%$, and $50 \%$ detection efficiency.

For all geometries, the detection efficiencies for the electromagnetic part are larger than the muonic ones for very vertical showers, but show the characteristic fall-off towards larger zenith angles, where the large atmospheric depth causes the electromagnetic part of the shower to die out, and only the muons remain. The kink visible in the muon distributions at $\cos (\theta) \approx 0.27$ appears because for larger zenith angles the shower core positions are outside the array and only the outer parts of the showers hit the instrumented area. Similarly, the stripes in the IceVeto detection efficiencies are caused by the shower positions coinciding with the concentric rings of detector stations. 
The IceVeto detection efficiencies exhibit another kink at very small zenith angles $(\cos (\theta) \approx 0.95)$; this is where IceTop with its denser instrumentation becomes responsible for the shower detection. Overall, the IceVeto geometry becomes efficient from muons at primary energies above $10 \mathrm{PeV}$. The higher instrumentation density of the $125 \mathrm{~m}$ grid detector shifts the whole distribution towards lower energies by about one order of magnitude, and only the $200 \mathrm{~m}$ grid detector with its fill factor of $1000 \mathrm{~m}^{2} \mathrm{~km}^{-2}$ is more than $99 \%$ efficient over almost the whole parameter space studied. If high detection efficiencies at energies below $1 \mathrm{PeV}$ are desired, a dense intrumentation is needed.

\section{CORSIKA-based MonteCarlo Simulation}

As discussed in the introduction, one advantage of a detailed simulation of air showers and detector response is the possibility to study rare phenomena. With a proton-air interaction length of about $70 \mathrm{~g} \mathrm{~cm}^{2}$, a fraction of $10^{-3}$ primary protons survive to a depth of $490 \mathrm{~g} \mathrm{~cm}^{2}$. Compare that to the roughly $690 \mathrm{~g} \mathrm{~cm}^{2}$ vertical depth of South Pole. Deep showers develop in a significantly denser atmosphere than average showers and hit the ice within a few interaction lengths of the first interaction. This reduces the probability that high-energy pions decay, and modifies the number and energy spectrum of the muons in the air shower. Conversely, primaries that interact high in the atmosphere develop on a less dense medium than average. Understanding the effects of these shower-to-shower fluctuations requires a detailed simulation of the cascading process.

In this study, 23000 proton-induced air showers were simulated with energies between $10 \mathrm{TeV}$ and $5 \mathrm{PeV}$ and zenith angles less than $65^{\circ}$. We used CORSIKA with the Sibyll 2.1 high-energy interaction model [14]. The simulation included a simplified model of the detector response with various detector array configurations. We have considered an array of scintillation detectors, each one consisting of one $1 \mathrm{~cm}$ thick polypropylene scintillator panel of varying surface area laid flat on to the surface of the ice. The signal recorded at each scintillation detector is proportional to the energy loss in the detector according to Bethe's formula. The signal is expressed in units of Vertical Equivalent Muon (VEM), defined as the signal deposited by a $3 \mathrm{GeV}$ muon crossing the detector vertically. With this convention, the threshold for recording a signal was set to 0.3 VEM. The flexibility of our setup will allow us to replace this simulation by a more detailed one in the future. In order to understand the effect of deep air showers, we simulated a small set of 2000 quasi-vertical $\left(\theta<20^{\circ}\right)$ proton-induced showers where the primary was forced to interact at points evenly distributed in depth between 0 and $500 \mathrm{~g} \mathrm{~cm}^{2}$.

\subsection{Results}

The air shower detection efficiency can also be expressed as a passing fraction, the fraction of air showers that do not trigger the veto array. This is shown in Figs. 5a and 5b in the case of four representative configurations and for vertical/inclined air showers. Using a simple scaling model, one expects the veto threshold to be proportional to the square of the array spacing and inversely proportional to the scintillator surface area. In other words, the fill factor described above. This implies that there is a trade-off between detector size and array spacing, but this is true only if the array spacing is comparable to the typical air shower footprint size. This can be seen in Fig. 5a, where decreasing array spacing and scintillator area while keeping the total fill factor constant produces a decrease in the passing fraction (light/dark blue points in Fig.5a). This is related to 


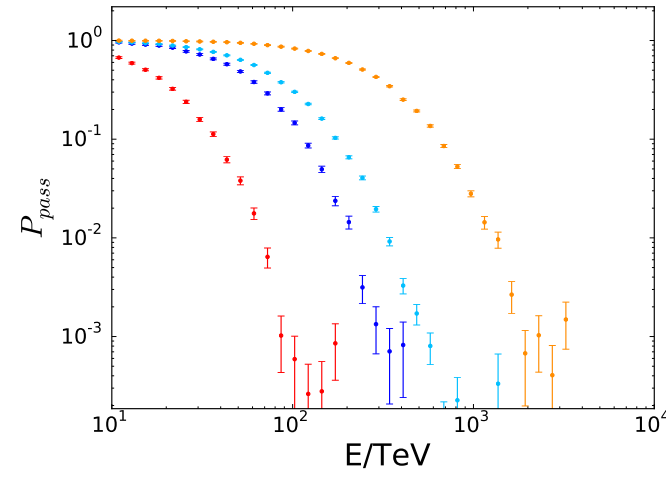

(a) $\theta<25^{\circ}$

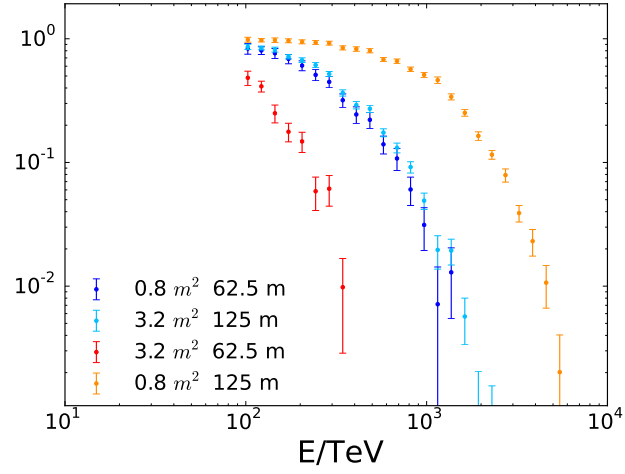

(b) $53^{\circ}-56^{\circ}$.

Figure 5: Passing fraction of quasi-vertical air showers $\left(\theta<25^{\circ}\right)$ and of showers arriving between $53^{\circ}$ and $56^{\circ}$. We show array configurations that have a spacing of $62.5 \mathrm{~m}$ or $125 \mathrm{~m}$ and a scintillator surface area of $0.8 \mathrm{~m}^{2}$ or $3.2 \mathrm{~m}^{2}$. The light and dark blue sets of points are configurations with equal fill factor, the change in detector spacing being offset by a change in scintillator area. The fill factors for these configurations are $9.5 \times 10^{-4}, 2.4 \times 10^{-4}$ and $5.9 \times 10^{-5}$. For a discussion see text.

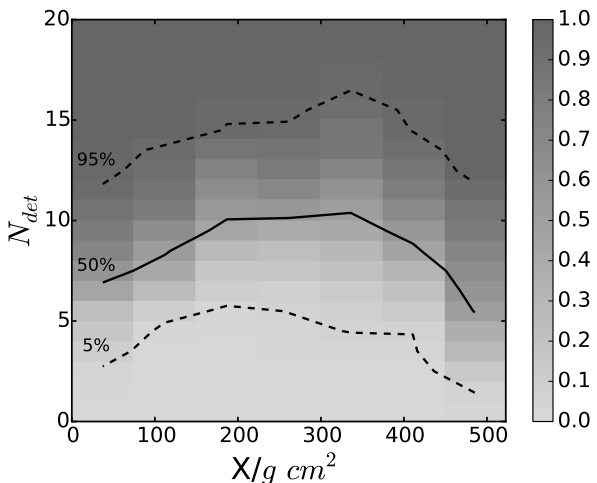

(a) $100 \mathrm{TeV}$

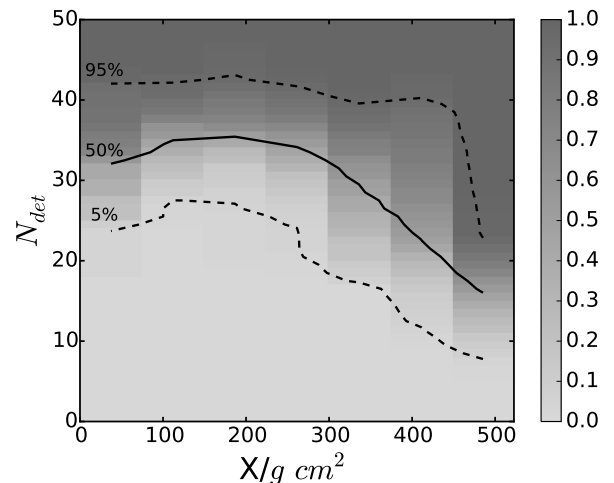

(b) $800 \mathrm{TeV}$

Figure 6: Fraction of air showers with number of detectors with signal smaller than $N_{d e t}$, as a function of the depth of first interaction of the primary cosmic ray X. The lines correspond to the $5 \%, 50 \%$ and $95 \%$ quantiles. Two primary energies are displayed: $100 \mathrm{TeV}$ (left) and $800 \mathrm{TeV}$ (right). Both correspond to $3.2 \mathrm{~m}^{2}$ scintillators in a regular triangular array with $62.5 \mathrm{~m}$ spacing.

the fact that the detection is dominated by the electromagnetic component of the air shower. By contrast, in Fig. 5b, where the air showers are dominated by the muon component, with a wider extent, the scaling holds.

The effect of the point of first interaction can be seen in Figs. 6a and 6b. The highest point of the curve corresponds to events in which the shower maximum lies at the surface. One can clearly see that deeper and shallower showers have a lower number of detectors registering a signal. At $800 \mathrm{TeV}$, the fraction of showers not detected by the veto are mostly produced at large depths, while at $100 \mathrm{TeV}$ some of them are produced at small depths. This is for quasi-vertical air showers, but one expects a similar behavior for inclined air showers. 
The passing fraction was estimated down to values around $10^{-3}$, as seen in Fig. 5. In order to probe smaller values, more efficient ways of generating simulated datasets are needed. The passing fraction is determined mostly by the primary point of first interaction, as shown in Fig. 6. It is also affected by the subsequent development of the air shower. We are working on algorithms that exploit this fact in order to efficiently simulate showers that have a small probability to be detected by the surface veto.

\section{Summary}

There are qualitatively two distinct regimes for a surface veto: vertical $(\cos (\theta) \gtrsim 0.5)$ and inclined $(\cos (\theta) \lesssim 0.5)$ (Fig. 4). Focusing on one or the other leads to different detector design decisions. In the vertical case, the threshold is determined by the sensitivity to the electromagnetic component of the air shower, and it depends on the snow accumulation. In this case, thresholds as low as $100 \mathrm{TeV}$ seem to be attainable with a fill factor of $10^{-3}$, and reasonable array spacing and size (5). In the inclined case, the threshold depends on the sensitivity to muons and it is larger by about a factor of 4 at $55^{\circ}$. In both cases a detailed study must be done in order to estimate the energy at which the detectors reach a passing fraction of $10^{-4}$ to $10^{-6}$. A detailed simulation of air showers and the in-ice detector response are needed to understand the effect of rare events.

\section{References}

[1] IceCube Collaboration, IceVeto: An Extension of IceTop to Veto Horizontal Air Showers, in Proc. of the 33rd ICRC \#374, Rio de Janeiro, Brasil, 2013. arXiv:1309. 7010.

[2] IceCube Collaboration, M. G. Aartsen et al., Science 342 (2013) 1242856, [arXiv: 1311. 5238].

[3] IceCube Collaboration, R. Abbasi et al., Nucl.Instrum.Meth. A700 (2013) 188-220, [arXiv:1207.6326].

[4] IceCube Collaboration, A search for extremely high energy neutrinos in 6 years of IceCube data, PoS(ICRC2015)1064 these proceedings.

[5] IceCube Collaboration, Results of neutrino point source searches with 2008-2014 IceCube data above $10 \mathrm{TeV}$, PoS(ICRC2015)1047 these proceedings.

[6] IceCube Collaboration, IceTop as Veto for IceCube, PoS(ICRC2015)1086 these proceedings.

[7] IceCube Collaboration, The IceCube-Gen2 High Energy Array, PoS(ICRC2015)1146 these proceedings.

[8] IceCube Collaboration, Motivations and Techniques for a Surface Detector to Veto Air Showers for Neutrino Astronomy withIceCube in the Southern Sky, PoS(ICRC2015)1156 these proceedings.

[9] D. Heck, G. Schatz, T. Thouw, J. Knapp, and J. N. Capdevielle, FZKA-6019 (1998).

[10] T. K. Gaisser, Cosmic Rays and Particle Physics. Cambridge University Press, 1991.

[11] K. Greisen, Ann.Rev.Nucl.Sci 10 (1960) 63-108.

[12] IceCube Collaboration, Surface Muons in IceTop, PoS(ICRC2015)267 these proceedings.

[13] IceCube Collaboration, Studying Cosmic Ray Composition with IceTop using Muon and Electromagnetic Lateral Distributions, PoS(ICRC2015)338 these proceedings.

[14] E. J. Ahn, R. Engel, T. K. Gaisser, P. Lipari and T. Stanev, Phys. Rev. D 80 (2009) 094003 